\title{
Numerical study of suspensions of nucleated capsules at finite inertia
}

\author{
Arash Alizad Banaei, Armin Shahmardi, and Luca Brandt \\ Linné Flow Centre and SeRC (Swedish e-Science Research Centre), Department of Engineering Mechanics, \\ Royal Institute of Technology (KTH), S-100 44 Stockholm, Sweden
}

(Received 13 May 2019; accepted 15 March 2021; published 6 April 2021)

\begin{abstract}
We study the rheology of suspensions of capsules with a rigid nucleus at negligible and finite flow inertia by means of numerical simulations. The capsule membrane is modeled as a thin Neo-Hookean hyperelastic material and the nucleus as a rigid particle with radius equal to half the radius of the undeformed spherical capsules. The fluid and solid motion are coupled with an immersed boundary method, validated for both the deformable membrane and the rigid nucleus. We examine the effect of the Reynolds number, capillary number, and volume fraction on the macroscopic properties of the suspensions, comparing with the case of capsules without nuclei. To explain the rheological measurables, we examine the mean capsule deformation, the mean orientation with respect to the flow direction, and the stress budget. The results indicate that the relative viscosity decreases with the capillary number, i.e., increasing deformability, and increases with inertia. The presence of a nucleus always reduces the membrane deformation. Capsules align more in the flow direction at higher capillary numbers and at higher volume fractions, where we also see a significant portion of them oriented with their longer deformed axis in the spanwise direction. When increasing inertia, the alignment with the flow decreases while more capsules orient in the spanwise direction. The first normal stress difference increases with the capillary number and it is always less for the nucleated capsules. Finally, the relative viscosity and the first normal stress difference increase with the capsule volume fraction, an effect more pronounced for the first normal stress difference.
\end{abstract}

DOI: 10.1103/PhysRevFluids.6.044301

\section{INTRODUCTION}

The rheological properties of capsule suspensions have great importance in medical applications, as well as in process industry. Capsules are defined here as droplets of a second fluid enclosed by a thin elastic membrane, transported and flowing in a carrier phase. Typically, capsules conserve volume while, unlike vesicles, their surface area can vary during the flow as a result of flow-induced deformations. As a classic example, because of the presence of the deformable red blood cells, blood is typically modeled as a suspension of deformable capsules and therefore characterized by a non-Newtonian viscoelastic behavior. The rheological properties are highly correlated to the membrane elasticity and to cell-cell interactions in denser conditions; see, among others, Reasor et al. [1]. In particular, the two main distinctive characteristics of the viscoelastic behavior - the relative viscosity as a function of the applied shear and the normal stresses-have been found to depend both on the average capsule deformation and on the capsule orientation angle; see, e.g., the recent

Published by the American Physical Society under the terms of the Creative Commons Attribution 4.0 International license. Further distribution of this work must maintain attribution to the author(s) and the published article's title, journal citation, and DOI. Funded by Bibsam. 
study by Matsunaga et al. [2]. Here, we study capsule suspensions as a model of cells in simple shear flows including the inertial regime and considering an additional complexity: the presence of a rigid nucleus. As shown here, the global suspension behavior is affected by the presence of the rigid nucleus inside the capsules, which we use to model, as examples, malaria-infected blood cells or eukaryotic cancer cells [3].

An expression for the relative viscosity of capsule suspensions has been derived analytically by Barthes-Biesel and Chhim [4] in the limit of small capillary numbers, predicting a shear-thinning behavior for dilute suspensions. The rheology of concentrated suspensions of viscoelastic particles was studied by Snabre and Mills [5] using an analytical Kelvin-Voigt model. These authors report shear thinning at high volume fractions while the effective viscosity was found to be almost independent of the shear rate at lower volume fractions. With regard to numerical studies, a coupled lattice-Boltzmann and finite-element method was proposed by MacMeccan et al. [6] for the simulation of deformable particles, enabling efficient simulations of suspensions at high volume fractions. These authors consider initially spherical and biconcave capsule suspensions at $40 \%$ volume fraction, and they report a significantly lower viscosity for initially spherical capsule suspensions, both suspensions displaying shear-thinning behavior.

The rheology of dilute capsule suspensions has been studied using three-dimensional numerical simulations by Bagchi and Kalluri [7], where the role of the viscosity contrast between the fluid inside and outside of the capsules is also examined. These authors observe shear thinning and a nonmonotonic variation of the relative viscosity with the viscosity ratio. The effect of the swinging and tumbling motions of initially oblate capsules on the rheological properties of dilute suspensions is investigated in Ref. [8], again by means of numerical simulations, showing that unlike spherical capsule suspensions, the system response is clearly time-dependent while the time-averaged rheological properties are qualitatively similar to those of suspensions of spherical capsules. A numerical study of the dynamics and microstructure of dense capsule suspensions is presented by Clausen et al. [9]; those authors find that the first normal stress difference undergoes a sign change at relatively small deformations. Dense suspensions of red blood cells have been investigated numerically in Ref. [1], where it was found that the viscosity is dependent on the orientation and bending modulus of the cells and that the normal stress tensor is characterized by a transition from compressive to tensile states in the flow direction when increasing the shear rate. In addition, it is shown that the bending stiffness and the initial shape of the capsules have a significant effect on the first normal stress difference. Krüger et al. [10] investigated the role of flow inertia on the rheology of semidilute capsule suspensions with a finite-element immersed-boundary lattice-Boltzmann solver, concluding that for low capillary numbers there is a monotonic increase of the shear viscosity with the flow inertia, whereas for larger capillary numbers the viscosity first increases and then decreases when further increasing the flow inertia.

The behavior of dense biconcave capsule suspensions is studied numerically by Gross et al. [11] by varying the volume fraction up to $90 \%$. These authors observe very large viscosity and first normal stress differences at high volume fractions while the suspensions maintain their shear thinning behavior; the Herschel-Bulkley rheological curves are used to fit these data. A multiscale numerical study on blood flow was performed by Fedosov et al. [12], who considered red blood cell suspensions in tubes with different diameters and reported a large increase of the relative viscosity when decreasing the tube diameter. Recent theoretical and numerical results on the rheological behavior of polymeric semi-dilute soft colloid, vesicle, capsule, and cell suspensions are reviewed in Ref. [13], which mentions, among other things, that the viscosity is much larger for aggregating cell suspensions than for nonaggregating cells. Matsunaga et al. [2] recently performed an extensive numerical study on the rheology of dense suspensions of initially spherical capsules with volume fractions up to $40 \%$. They observe that unlike rigid spheres, the relative viscosity increases almost linearly with the volume fraction even at high volume fractions in the case of deformable capsules. In addition, the capsule deformation increases when increasing the volume fraction while the mean capsule angle with respect to flow direction decreases, and this reduction causes larger stress anisotropy, resulting in larger first normal stress differences. Rosti et al. [14,15] 
have investigated suspensions of deformable particles in the case of negligible inertia, reporting an increase of viscosity with the particle volume fraction and shear thinning with deformability, i.e., increasing the capillary number. In addition, they report a peak of the first normal stress difference at moderate capillary numbers. The data for the effective viscosity are shown to collapse to a universal function by defining an effective volume fraction lower than the nominal one due to the particle deformation. A similar scaling was confirmed by simulations of the rheology of deformable red blood cells in dilute, semidilute, and dense suspensions [16].

All works discussed so far have considered simple capsules, without any rigid structure inside. Malaria-infected red blood cells and eukaryotic cells, on the other hand, have a nucleus inside the external membrane, which can significantly affect the capsule dynamics in a flow. The dynamics of malaria-infected red blood cells have been studied in Ref. [17] by means of numerical simulations; the results show a large increase of the relative viscosity in a microcircular channel with a diameter of $12 \mu \mathrm{m}$ in the presence of a sick cell in the trophozoite stage, where the size of the nucleus is of the order of $20 \%$ of the cell. Spherical and nonspherical nucleated capsules in shear flow have also been investigated numerically by Luo et al. [18] and Luo and Bai [19], who found that the inner nucleus can significantly change the outer capsule dynamics, and that the size of the inner capsule, which is used to mimic a stiff nucleus, strongly affects the overall dynamics. Single nucleated capsules in shear flow are also simulated in Ref. [20], showing that the presence of the nucleus reduces the capsule deformation, and that inertial effects do not have significant effects on the deformation of isolated nucleated capsules. The motion of single nucleated capsules in a constricted microchannel has been studied recently by Gounley et al. [3], who also report lower deformation for nucleated capsules. All of these studies focus on single cells; no studies on the behavior of the nucleated cell suspensions are available as of yet.

The goal of the present study is therefore to extend our previous work on the dynamics of a single nucleated capsule [20] to suspensions of initially spherical capsules with nucleus and study the rheological behavior and the capsule deformation at negligible and finite flow inertia, comparing the results to those pertaining to suspensions of simple capsules. The same numerical setup used in Ref. [20], where the nucleus is considered as a rigid particle, will be employed. The ratio between the nucleus and the undeformed capsule diameter will be kept constant while examining the role of the capillary number, Reynolds number, and volume fraction on the relative viscosity, first normal stress difference, mean deformation, mean orientation, and elastic energy of simple and nucleated capsule suspensions; in particular, the relationship between the rheological properties and the deformation and orientation angle of the capsules will be discussed.

\section{GOVERNING EQUATIONS AND NUMERICAL METHOD}

The geometrical configuration adopted is depicted in Fig. 1. We consider a suspension of $N$ initially spherical capsules of radius $R$ in a shear flow with top and bottom walls moving in opposite directions imposing a shear rate $\dot{\gamma}$. The fluids inside and outside the capsules have the same viscosity $\mu$ and density $\rho$ in order to focus on the effect of elasticity and inertia. In the case of nucleated capsules, the nucleus is modeled as a rigid spherical particle of radius $0.5 R$, initially located at the center of each capsule and free to move inside it.

\section{A. Governing equations}

The dynamics of an incompressible Newtonian fluid are governed by the momentum and continuity equations:

$$
\frac{\partial}{\partial x_{i}}\left(u_{i}\right)=0, \quad \frac{\partial u_{i}}{\partial t}+u_{j} \frac{\partial u_{i}}{\partial x_{j}}=-\frac{\partial P}{\partial x_{i}}+\frac{1}{\operatorname{Re}} \frac{\partial^{2} u_{i}}{\partial x_{j}^{2}}+f_{i},
$$




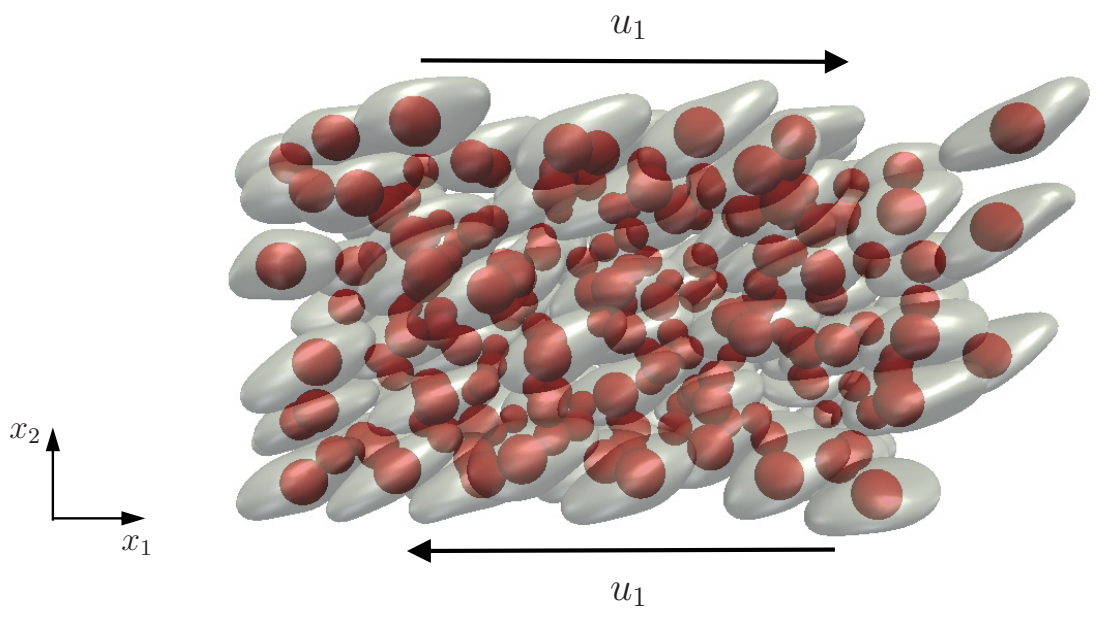

FIG. 1. Nucleated capsules in shear flow. The visualization refers to a suspension with volume fraction $\phi=31.42 \%$.

where $u_{i}$ is the velocity vector, $P$ is the hydrodynamic pressure, $\operatorname{Re}=\frac{\rho \dot{\gamma} R^{2}}{\mu}$ is the Reynolds number, with $R$ the initial radius of the capsules, $\tau_{i j}$ the fluid shear stress tensor, and $f_{i}$ represents here the elastic forces arising from the interactions with the capsule membrane.

\section{B. Capsule dynamics}

The capsule membrane is modeled as a hyperelastic two-dimensional material, i.e., with thickness much smaller than the capsule initial radius, and the same density as the fluid, which implies that the relative acceleration of the membrane with respect to fluid is negligible. A fixed Cartesian coordinate is considered for the fluid and capsule motion while a moving curvilinear coordinate system is needed to represent the capsule deformation. As shown in Fig. 2, the fixed Cartesian coordinates $\left(x_{1}, x_{2}, x_{3}\right)$ are defined by the Cartesian based vectors $\left(e_{1}, e_{2}, e_{3}\right)$, whereas the second coordinate system $\left(\xi^{1}, \xi^{2}\right)$, which represents the points on the surface of the capsule, is a local covariant base $\left(a_{1}, a_{2}, a_{3}\right)$. Each point on the capsule surface is represented in both coordinate systems, $\boldsymbol{X}\left(\xi^{1}, \xi^{2}\right)=\left(x_{1}, x_{2}, x_{3}\right)$.

Two of the covariant base vectors are tangential to the curves of constant $\xi^{\alpha}, a_{\alpha}=X_{\alpha}, \alpha=1,2$, with the third normal to the first two base vectors. Here, the subscript $\alpha$ indicates derivative with respect to $\xi^{\alpha}$. Also, in this study, vectors and metric tensors in the reference state are shown in capital letters. Assuming $\xi^{1}$ and $\xi^{2}$ as the latitudinal and longitudinal angles on the surface of the

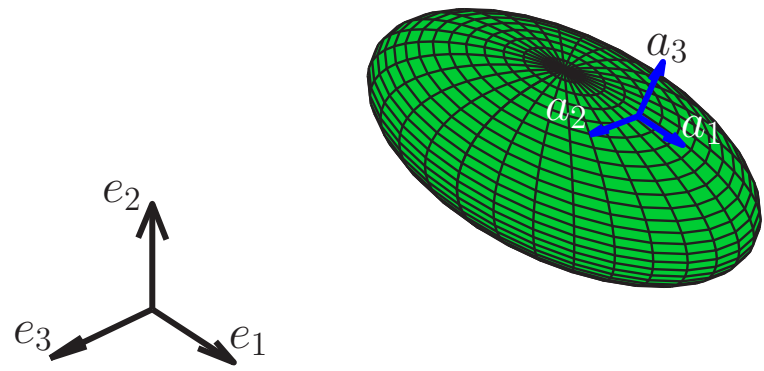

FIG. 2. Schematic of a deformed capsule and Cartesian and curvilinear base vectors. 
capsule, one can write the three covariant base vectors as

$$
a_{1}=\frac{\partial \boldsymbol{X}}{\partial \xi^{1}}, \quad a_{2}=\frac{\partial \boldsymbol{X}}{\partial \xi^{2}}, \quad a_{3}=\frac{a_{1} \times a_{2}}{\left|a_{1} \times a_{2}\right|} .
$$

The contravariant base vectors, $\left(a^{1}, a^{2}, a^{3}\right)$, are

$$
a^{\alpha} a_{\beta}=\delta_{\beta}^{\alpha},
$$

where $\delta_{\beta}^{\alpha}$ is the Kronecker delta. Any arbitrary vector $\boldsymbol{U}$ can be written in both coordinates,

$$
\boldsymbol{U}=u^{i} \boldsymbol{a}_{i}=u_{i} \boldsymbol{a}^{i}=\boldsymbol{U}_{\boldsymbol{x}_{i}} \boldsymbol{e}_{i},
$$

where $\boldsymbol{x}_{i}$ stands for the Cartesian components. The metric tensors are defined as

$$
a_{\alpha \beta}=\boldsymbol{a}_{\alpha} \cdot \boldsymbol{a}_{\beta}, \quad a^{\alpha \beta}=\boldsymbol{a}^{\alpha} \cdot \boldsymbol{a}^{\beta} .
$$

The deformation gradient tensor is defined as $\mathbf{F}=\mathbf{a}_{\alpha} \otimes \mathbf{A}^{\alpha}$, and consequently the GreenLagrange strain tensor is $\mathbf{e}=\left(\mathbf{F}^{T} \cdot \mathbf{F}-\mathbf{I}\right) / 2$. The invariants of the transformation are

$$
I_{1}=\lambda_{1}^{2}+\lambda_{2}^{2}-2, \quad I_{2}=\lambda_{1}^{2} \lambda_{2}^{2}-1=J_{s}^{2}-1,
$$

where $\lambda_{1}$ and $\lambda_{2}$ are the principal stretch ratios. The membrane is assumed here to behave as a Neo-Hookean material, whose strain energy $\boldsymbol{W}_{s}^{\mathrm{NH}}$ is defined in nondimensional form as a function of the deformation invariants as

$$
W_{s}^{\mathrm{NH}}=\frac{1}{2 \operatorname{ReCa}}\left(I_{1}-1+\frac{1}{I_{2}+1}\right),
$$

where $\mathrm{Ca}$ is the capillary number defined as the ratio of viscous forces to elastic forces,

$$
\mathrm{Ca}=\frac{\mu \dot{\gamma} R}{G_{s}},
$$

with $G_{s}$ the surface shear modulus. The Cauchy stress tensor $\boldsymbol{T}$, the elastic stress, can be obtained from the strain energy:

$$
T^{\alpha \beta}=\frac{2}{\lambda_{1} \lambda_{2}} \frac{\partial \boldsymbol{W}_{s}^{\mathrm{NH}}}{\partial I_{1}} \boldsymbol{A}^{\alpha \beta}+2 \lambda_{1} \lambda_{2} \frac{\partial \boldsymbol{W}_{s}^{\mathrm{NH}}}{\partial I_{2}} \boldsymbol{a}^{\alpha \beta} .
$$

The deformation of a neutrally buoyant membrane (no density difference with the fluid), for which inertia can be neglected, is determined by the local equilibrium between the external load and the elastic stresses:

$$
\nabla_{s} \boldsymbol{T}+\boldsymbol{q}=\mathbf{0},
$$

where $\nabla_{s}$ is the surface divergence operator. To express this equation in curvilinear coordinates, we write the load vector as $\boldsymbol{q}=q^{\beta} \boldsymbol{a}_{\beta}+q^{n} \boldsymbol{a}_{n}$, and we decompose Eq. (10) into tangential and normal components:

$$
\frac{\partial T^{\alpha \beta}}{\partial \xi^{\alpha}}+\Gamma_{\alpha \lambda}^{\alpha} T^{\lambda \beta}+\Gamma^{\beta} \alpha \lambda T^{\alpha \beta}+q^{\beta}=0, \beta=1,2, \quad T^{\alpha \beta} b_{\alpha \beta}+q^{n}=0,
$$

where $\Gamma_{\alpha \lambda}^{\beta}=\boldsymbol{a}_{\alpha, \lambda} \boldsymbol{a}^{\beta}$ is the Christoffel symbol. In the case of a membrane with nonzero bending stiffness, the bending moment and shear tension should also be considered [20,21].

This is incorporated into the model using a linear isotropic model for the bending moment as a function of deformation [21,22]:

$$
\mathbf{M}_{\beta}^{\alpha}=\frac{-\tilde{B}}{W e}\left(b_{\beta}^{\alpha}-B_{\beta}^{\alpha}\right),
$$


where $\tilde{B}=\frac{G_{b}}{G_{s} R^{2}}$ is the nondimensional bending modulus and $b_{\beta}^{\alpha}=\boldsymbol{a}_{\alpha, \beta} \cdot \boldsymbol{n}$ is the second fundamental form of the surface ( $B_{\beta}^{\alpha}$ refers to that of the reference configuration). According to the local torque balance, including bending moments on the membrane, the transverse shear vector $\mathbf{Q}$ and in-plane stress tensor $\mathbf{T}$ can be obtained as

$$
M_{\mid \alpha}^{\alpha \beta}-Q^{\beta}=0, \quad \boldsymbol{\varepsilon}_{\alpha \beta}\left(T^{\alpha \beta}-b_{\gamma}^{\alpha} M^{\gamma \beta}\right)=0,
$$

where ' $\mid \alpha$ ' represents the covariant derivative, and $\varepsilon$ is the two-dimensional Levi-Civita tensor. The left-hand side of Eq. (12) identifies the antisymmetric part of the in-plane stress tensor, which is always zero as proven in Ref. [23]. Including the transverse shear stress $Q$, the local stress equilibrium, including bending, finally gives

$$
\frac{\partial T^{\alpha \beta}}{\partial \xi^{\alpha}}+\Gamma_{\alpha \lambda}^{\alpha} T^{\lambda \beta}+\Gamma_{\alpha \lambda}^{\beta} T^{\alpha \lambda}-b_{\alpha}^{\beta} Q^{\alpha}+q^{\beta}=0, \quad \beta=1,2, \quad T^{\alpha \beta} b_{\alpha \beta}-Q_{\mid \alpha}^{\alpha}+q^{n}=0 .
$$

\section{Numerical method}

The flow equations are solved by a fractional step method on a uniform grid; see more details in Refs. [20,24] and in previous studies on suspensions of rigid particles [24-26]. The momentum equations are solved by the finite volume method using central differences for the spatial discretization. For the temporal integration, the Adams-Bashforth scheme is used for the convective terms while the diffusive terms are treated implicitly. The reader is referred to our methodology paper [20] for further details.

To solve the fluid solid interactions, we use the immersed boundary method (IBM), which was first introduced by Peskin [27]. Since then, different IBM approaches have been proposed, suited for simulating different physical problems; see, e.g., the review by Mittal and Iaccarino [28]. In this study, the original method proposed by Peskin [27] is implemented for the capsule motion, while a direct forcing IBM is used for the rigid particles [24]. As in a classic IBM, two sets of grid points are considered, namely an Eulerian and a Lagrangian grid, which are linked by the fluid-solid interaction force, corresponding to the elastic stresses in our case. As the details are provided in Ref. [20], we report here directly the solution steps, which can be summarized as follows:

(i) Solve the flow field on the Eulerian mesh.

(ii) Interpolate the solution velocity field onto the Lagrangian grid points, discretizing Eq. (14) using the approximated Dirac delta function, $\delta$, first proposed by Roma et al. [29],

$$
\boldsymbol{U}_{i \boldsymbol{b}}(\boldsymbol{X}, \boldsymbol{t})=\int_{\Omega} \boldsymbol{u}(x, t) \delta(\boldsymbol{X}-x) d x,
$$

where $x$ and $X$ are the Eulerian and Lagrangian coordinates.

(iii) Calculate the tangential and normal vectors on the Lagrangian elements and the elastic force per unit area by solving for the membrane dynamics.

(iv) Spread the elastic force onto the Eulerian mesh by the same Dirac $\delta$ function proposed by Roma et al. [29],

$$
\boldsymbol{f}(X, t)=\int-\boldsymbol{q}(\boldsymbol{X}, t) \delta(x-\boldsymbol{X}) d s .
$$

(v) Solve the Navier-Stokes equations for the flow field, updating the fluid-structure interaction force.

(vi) Update the positions of the Lagrangian points,

$$
\boldsymbol{X}^{n+1}=\boldsymbol{X}^{n}+\boldsymbol{U}_{i b}^{n} \Delta t
$$

where $\Delta t$ is the time step.

These operations are performed each time step. Following the work by Pranay et al. [30], an extra displacement correction is imposed on the Lagrangian points at every time step to ensure the 
volume conservation for the capsules,

$$
z=\frac{-3\left(V-V_{0}\right)}{A} \boldsymbol{n}
$$

where $V$ and $V_{0}$ are the instantaneous and initial volumes, $A$ is the capsule surface area, and $\boldsymbol{n}$ is the unit normal to the capsule surface area.

To compute the elastic stresses, derivatives on the capsule surface are needed; for an accurate discretization, the capsule shape is represented by a truncated series of spherical harmonic functions,

$$
x\left(\xi^{1}, \xi^{2}\right)=\sum_{n=0}^{N_{\mathrm{SH}}-1} \sum_{m=0}^{n} \bar{P}_{n}^{m}\left(\cos \xi^{1}\right)\left[a_{n m} \cos \left(m \xi^{2}\right)+b_{n m} \sin \left(m \xi^{2}\right)\right],
$$

with $N_{\mathrm{SH}}^{2}$ the total number of spherical harmonics. $\bar{P}_{n}^{m}(x)$ is the normalized Legendre polynomial,

$$
\bar{P}_{n}^{m}(x)=\frac{1}{2^{n} n !} \sqrt{\frac{(2 n+1) n-m) !}{2(n+m) !}}\left(1-x^{2}\right)^{\frac{m}{2}} \frac{d^{n+m}}{d x^{n+m}}\left(x^{2}-1\right)^{n} .
$$

In spherical coordinates, the domain is discretized on a quadrilateral grid using Gauss-Legendre (GL) quadrature intervals in $0 \leqslant \xi^{1} \leqslant \pi$ and uniform spacing in $0 \leqslant \xi^{2} \leqslant 2 \pi$.

In the case of nucleated capsules, we model the nucleus as a rigid particle using the directforcing immersed boundary method proposed by Breugem [31] and later used, among others, in Refs. [24,31,32]. Further details are therefore not provided here.

To avoid capsule-capsule, capsule-particle, and capsule-wall overlapping, needed at finite Reynolds numbers, we use a repulsive force in the form of a Morse potential [33]. This is activated when the distance between two surfaces becomes lower than two grid cells. The general form of the potential is

$$
\zeta=D_{e}\left[e^{-2 a\left(r_{L}-r_{e}\right)}-2 e^{-a\left(r_{L}-r_{e}\right)}\right]
$$

where $D_{e}$ is the interaction strength, $a$ is a geometrical scaling factor, $r_{L}$ is the distance between two surfaces, and $r_{e}$ is the zero cutoff force distance, which is two grid cells here. The repulsive force is the derivative of the potential $\boldsymbol{F}=-\frac{\partial \zeta}{\partial r} \boldsymbol{d}_{r}$, where $\boldsymbol{d}_{r}$ is the unit vector between the two surfaces. The contact force computed at each Lagrangian point is spread to the Eulerian mesh and added in the momentum equation, as shown in Eq. (15).

\section{PROBLEM DESCRIPTION AND CODE VALIDATION}

\section{A. Problem description}

To study the rheology of the nucleated and denucleated capsules, we consider the steady shear flow between two moving walls. The dimensions of the channel in the streamwise, wall normal, and spanwise directions are $16 \times 10 \times 16$ in units of the undeformed capsule radius $R$, following Refs. [34,35]. The flow is assumed to be periodic in the streamwise and spanwise direction, whereas the upper and lower wall have the same velocity magnitude and opposite direction, a classic Couette flow.

The domain is discretized with $256 \times 160 \times 256$ grid points in the streamwise, wall-normal, and spanwise directions, respectively. The capsule surface is typically represented with $36 \times 72$ points in the latitudinal and longitudinal directions, corresponding to 2592 Lagrangian points on each capsule surface and to 1296 spherical harmonics and 36 modes to resolve the capsule deformation. For a few cases, those exhibiting small deformations, i.e., low capillary and Reynolds numbers, we have used $24 \times 48$ points as this is found to be sufficient to resolve the capsule surface. The simulations have been performed for different Reynolds numbers, capillary numbers $\mathrm{Ca}=\frac{\mu \dot{\gamma} R}{G_{s}}$, and different volume fractions. We first consider 192 capsules corresponding to a volume fraction of $31.42 \%$, which is a relatively dense suspension where capsule-capsule interactions are important. 
The frequent capsule-capsule interactions require using time steps smaller than those for single capsules to be able to resolve the flow between the capsules. In a later section, the role of capsule volume fraction is discussed for some selected cases. We have considered both simple and nucleated capsules, with the viscosity of the fluid inside and outside of the capsules assumed to be equal.

In this study, we consider a finite bending stiffness of the membrane. To have an equal effect of bending stiffness for different capillary and Reynolds numbers, the bending stiffness is made nondimensional with the shear modulus

$$
\tilde{B}=\frac{G_{b}}{G_{s} R^{2}}
$$

For all cases presented below, the bending stiffness is set to $\tilde{B}=0.05$ except for the validation case, when we set $\tilde{B}=0.002$ for $\mathrm{Ca}=0.2, \tilde{B}=0.005$ for $\mathrm{Ca}=0.3$ and 0.4 , and $\tilde{B}=0.01$ for $\mathrm{Ca}=0.5$. These values are found to give stable solutions with a negligible effect on the capsule dynamics, and therefore they are used for comparisons with literature results obtained for nominally zero bending stiffness.

\section{B. Rheological measures and capsule observables}

To validate the code against literature data on capsule suspensions before analyzing the behavior of nucleated capsules in different flow conditions, we need to quantify the stresses in the suspensions. The bulk stress is the result of both fluid stresses and fluid-solid interaction forces. Considering a volume $V$ that contains a large amount of particles, each of volume $V_{0}$ and surface area $A_{0}$, such that the suspension can be assumed homogeneous, and assuming a statistically stationary state, Batchelor [36] derived an expression for the bulk stress in suspension,

$$
\Sigma_{i j}=\frac{1}{V} \int\left(\sigma_{i j}-\rho u_{i}^{\prime} u_{j}^{\prime}\right) d V .
$$

For a Newtonian fluid, Eq. (22) is rewritten in nondimensional form as [36]

$$
\underbrace{\frac{\Sigma_{i j}}{\mu \dot{\gamma}}}_{\text {Total bulk stress }}=\underbrace{\frac{\operatorname{Re}}{V} \int_{V-\Sigma V_{0}}\left\{-p \delta_{i j}+\mu\left(\frac{\partial u_{i}}{\partial x_{j}}+\frac{\partial u_{j}}{\partial x_{i}}\right)\right\} d V}_{\text {Contribution of local Newtonian fluid }}+\underbrace{\frac{\operatorname{Re}}{V} \sum \int_{V_{0}} \sigma_{i j} d V-\frac{1}{V} \int u_{i}^{\prime} u_{j}^{\prime} d V}_{\text {Particle contribution, } \Sigma_{i j}^{P}},
$$

where $u_{i}^{\prime} u_{j}^{\prime}$ is the fluctuation stress of the fluid, vanishing when inertia is negligible. In our simulations, it is found that the stress arising from the velocity fluctuations is negligible also at the highest Reynolds number considered, $O(10)$, thus the contribution of the particles to the total stress can be rewritten for our purposes as

$$
\underbrace{\frac{\Sigma_{i j}^{P}}{\mu \dot{\gamma}}}_{\text {Total particle stress }}=\underbrace{\frac{\operatorname{Re}}{V} \sum \int_{A_{0}} F_{M i} x_{j} d S}_{\text {Membrane contribution }}+\underbrace{\frac{\operatorname{Re}}{V} \sum \int_{V_{R}} F_{R i} x_{j} d V}_{\text {Rigid particle contribution }}
$$

where $\boldsymbol{F}_{M}$ denotes the elastic forces from the membranes, and $\boldsymbol{F}_{R}$ denotes the fluid-solid interaction forces from the rigid nucleus. The relative shear viscosity $\eta$ is defined as

$$
\eta=\frac{\mu_{\mathrm{eff}}}{\mu}=1+\frac{\Sigma_{i j}^{P}}{\mu \dot{\gamma}},
$$

where $\mu_{\text {eff }}$ is the effective viscosity of the suspension and $\dot{\gamma}$ is the shear rate.

The first normal stress difference, which can be regarded as a measure of the suspension viscoelasticity, is defined as the difference between the streamwise and wall-normal component 
of the normal stresses,

$$
N_{1}=\frac{\Sigma_{11}^{P}-\Sigma_{22}^{P}}{\mu \dot{\gamma}},
$$

whereas the second normal stress difference, $N_{2}$, is the difference between the wall-normal and spanwise component of the normal stresses,

$$
N_{2}=\frac{\Sigma_{22}^{P}-\Sigma_{33}^{P}}{\mu \dot{\gamma}} .
$$

To provide a quantitative measure of the capsule deformation, we will use the Taylor parameter. To compute it, we introduce the moment of inertia tensor for each capsule in suspension [37],

$$
S_{i j}= \begin{cases}\int_{V}\left(x_{k}^{2}+x_{p}^{2}\right) d v, & i=j ; k, p \neq i \\ \int_{V} x_{i} x_{j} d v, & i \neq j .\end{cases}
$$

Note that the density $\rho$ does not appear in Eq. (28) since it is in nondimensional form. Each capsule is approximated by an ellipsoid with the same principal moments of inertia as those computed from the deformed capsule [38]. This symmetric tensor has three orthogonal eigenvectors and three eigenvalues, $\lambda_{1}, \lambda_{2}, \lambda_{3}$. The semiaxes of the equivalent ellipsoid $a_{i, i=1,3}$ are simply computed by equating the eigenvalues with the principal moments of inertia of a spheroid,

$$
\frac{2}{5} V\left(a_{1}^{2}+a_{2}^{2}\right)=\lambda_{3}, \quad \frac{2}{5} V\left(a_{2}^{2}+a_{3}^{2}\right)=\lambda_{1}, \quad \frac{2}{5} V\left(a_{1}^{2}+a_{3}^{2}\right)=\lambda_{2},
$$

yielding three equations for the three unknown $a_{1}, a_{2}, a_{3}$. In Eq. (29), $V$ is the scaled volume of the capsule, $4 \pi / 3$. Considering the three possible pairs defined by these three semiaxes, we choose the maximum value of the ratio

$$
D=\max \left(\frac{a_{i}-a_{j}}{a_{i}+a_{j}}\right)
$$

to quantify the maximum elongation of the capsule.

The above-mentioned definition is not sufficient to quantify the deformation when the capsule shape becomes complex; therefore, in addition to the deformation parameter $D$, we present the area ratio

$$
\mathrm{AR}=\frac{A-A_{0}}{A_{0}}
$$

where $A$ and $A_{0}$ are the deformed capsule and the initial surface area.

Finally, we also compute the mean orientation angle with respect to the streamwise direction, $\theta$. This is based on the eigenvectors of the moment of inertia tensor, then averaged for all capsules in suspensions. In particular, we first define the unit vector $\mathbf{d}=\left(d_{1}, d_{2}, d_{3}\right)$ as the eigenvector of $\mathbf{S}$ corresponding to the minimum eigenvalue $\lambda_{\min }=\lambda_{3}$; this is associated with the direction of maximum elongation because, as defined in Eq. (28), the capsule volume is distributed closer to this axis. The orientation angle in the shear plane is then computed based on the streamwise and wall normal components of the elongation vector $\mathbf{d}$,

$$
\theta=\cos ^{-1}\left(\frac{d_{1}}{\sqrt{d_{1}^{2}+d_{2}^{2}}}\right)
$$

\section{Validation}

The code has been validated for the case of a single capsule in a shear flow by Alizad Banaei et al. [20]. For the simulations of suspensions, we compare our results for the relative viscosity at $\operatorname{Re}=0.1$ and particle volume fraction $\phi=30 \%$ to those in Ref. [2] for different capillary numbers. 
TABLE I. Comparison between the relative viscosity from the present simulations and the results by Matsunaga et al. [2], where our data are obtained with $\mathrm{Re}=0.1$.

\begin{tabular}{lcccr}
\hline \hline & \multicolumn{3}{c}{ Ca } \\
\cline { 2 - 5 } & 0.2 & 0.3 & 0.4. & 0.5 \\
\hline Present work & 1.940 & 1.825 & 1.750 & 1.670 \\
Simulations of Ref. [2] & 1.818 & 1.716 & 1.635 & 1.574 \\
\hline \hline
\end{tabular}

The numerical values, reported in Table I, display an acceptable agreement where the differences are within the ranges typically reported in the literature [35,39]. We attribute the differences to the different mathematical model (Stokes flow versus Navier-Stokes at vanishing Reynolds number) and the numerical approach for both fluid and solid solvers.

\section{NUCLEATED SUSPENSIONS}

We now examine the dynamics of suspensions of nucleated capsules for different Reynolds and capillary numbers, as well as volume fractions. Figure 3 displays four snapshots of the capsules in suspensions, once the statistically steady state has been reached, for the most deformable case, $\mathrm{Ca}=0.5$, and for different Reynolds numbers. The figure suggests more deformation at higher Reynolds number; moreover, the nucleated capsules exhibit overall lower deformations: they are thicker in the regions close to their nucleus while their membrane deformation is larger in the regions far from the nucleus, which is consistent with the results in Refs. [18-20] on the dynamics of single nucleated cells. It can be observed that at $\mathrm{Re}=0.1$, i.e., negligible inertia, most capsules have their major axis in the shear plane, whereas at $\operatorname{Re}=5$ there are several capsules with their major axis oriented in the spanwise direction. To quantify these observations and their role for the global

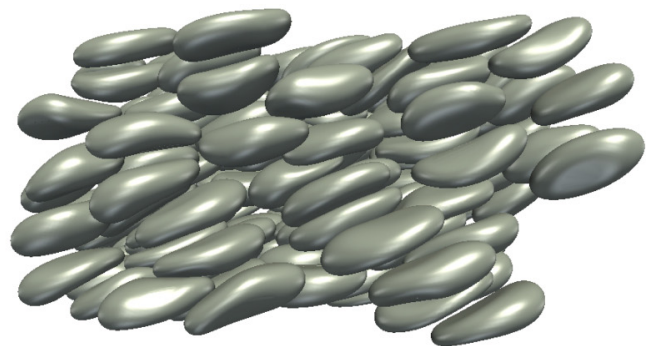

(a)

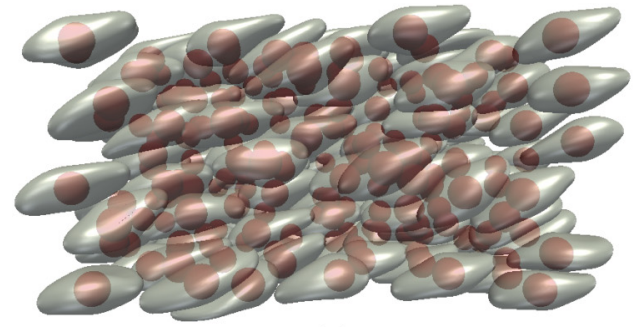

(c)

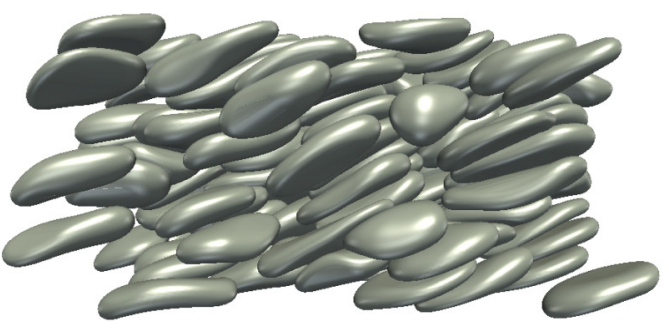

(b)

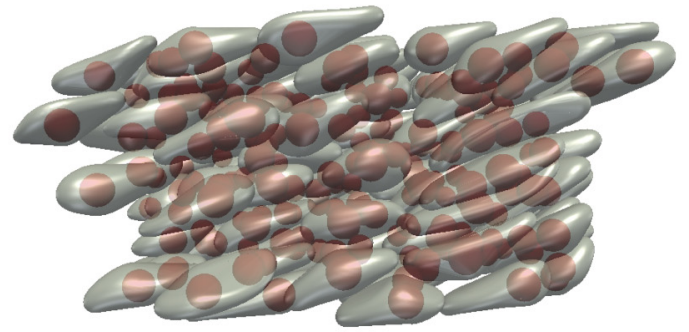

(d)

FIG. 3. Visualization of capsules with $\mathrm{Ca}=0.5$ and volume fraction $\phi=0.31$ in the flowing suspension at statistically steady state for the cases (a) $\operatorname{Re}=0.1$ without a nucleus, (b) $\operatorname{Re}=5$ without a nucleus, (c) $\operatorname{Re}=0.1$ with a nucleus, and (d) $\operatorname{Re}=5$ with a nucleus. 

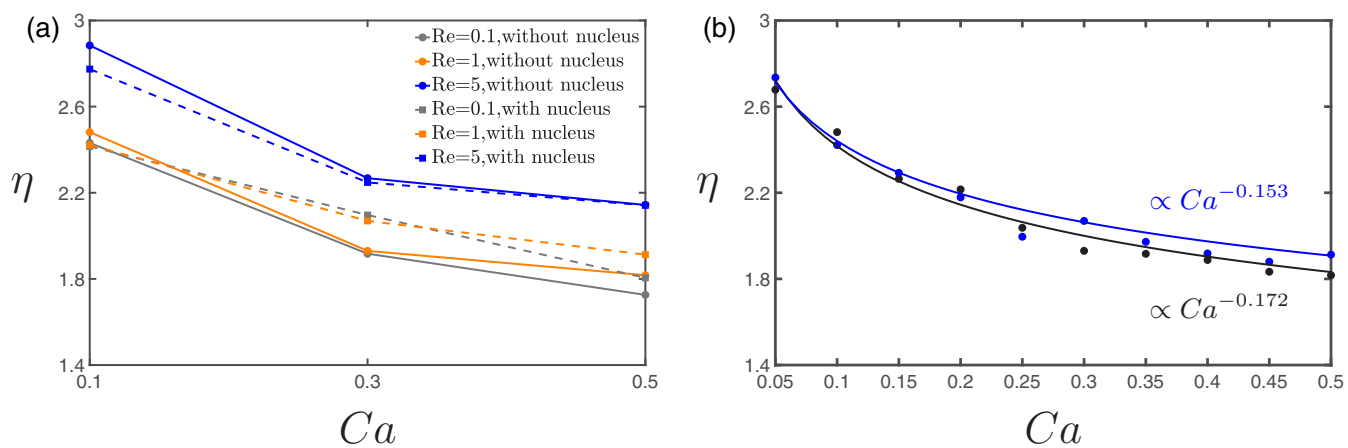

FIG. 4. (a) Relative viscosity of capsule suspensions as a function of the capillary number $\mathrm{Ca}$ and the Reynolds number Re for volume fraction $\phi=0.31$. Solid lines correspond to capsules without a nucleus, and the dashed line represents capsules with a rigid nucleus. Gray, orange, and blue pertain to cases with $\operatorname{Re}=0.1$, 1, and 5. (b) Relative viscosity of the capsule suspensions at $\mathrm{Re}=1$ with additional simulation points at different $\mathrm{Ca}$. Blue and black represent capsules with and without nucleus, respectively, and the corresponding solid line is a power-law fit to the data.

suspension behavior, the eight observables introduced in the previous section will be compared, namely the relative shear viscosity, the first normal stress difference, the orientation angle, the elastic energy, the Taylor parameter, the area ratio, the spanwise orientation, and moments of inertia. All the mean quantities are averaged over time and over capsules/particles.

\section{A. Effects of Reynolds number and capillary number at a fixed volume fraction}

We present in Fig. 4 the effect of the capillary number and the Reynolds number on the relative viscosity of simple and nucleated capsule suspensions. The results in Fig. 4(a) show that the relative viscosity decreases with the capillary number for both types of capsules; this shear thinning with deformability is in agreement with previous results for a capsule at negligible inertia and deformable hyperelastic particles $[2,11,14,15]$, as well as suspensions of flexible filaments $[40,41]$. The origin of this behavior will be further examined later when presenting the suspension stress budget.

To better quantify the observed shear thinning, we performed additional simulations with different capillary numbers at $\mathrm{Re}=1$; this is chosen as a compromise so that inertial effects are still limited, and computational time affordable. The results are presented in Fig. 4(b) with a fitted power-law curve to the data. The figure shows that the power-law behavior still holds in the presence of a nucleus inside the capsules. The variations with $\mathrm{Ca}$ decrease when increasing the capillary number, and the relative viscosity tends to approach a constant value, similarly to the limit at large shear rates of the viscosity curve of power-law fluids obeying a Carreau model.

As concerns the role of inertia, the data in Fig. 4(a) show that the relative viscosity increases with the Reynolds number, which we explain with a larger drag force on the capsules. The increase is more pronounced from $\mathrm{Re}=1$ to 5 . In the presence of a nucleus inside the capsule, an additional effect comes into play, namely the stresses induced by the nucleus. Figure 4 shows that at lower inertia, $\operatorname{Re} \leqslant 1$, the presence of the nucleus increases the relative viscosity, which is shown more clearly in Fig. 4(b). At $\mathrm{Re}=5$, however, the difference between the cases with and without a nucleus is very small. This is because at higher inertia, the nucleus highly reduces the capsule deformation (as shown later in Fig. 7), which tends to reduce the relative viscosity [2], balancing the extra stress due to the nucleus itself.

To better understand the capsule deformation and to provide an indication of the distribution variance within the suspensions, we present in Fig. 5 the probability distribution function (PDF) of the equivalent ellipsoid semiaxes divided by their minimum value, $a_{j} / a_{\min }$, obtained once the suspension flow has reached the statistically steady state. The largest of two ratios can be seen as 

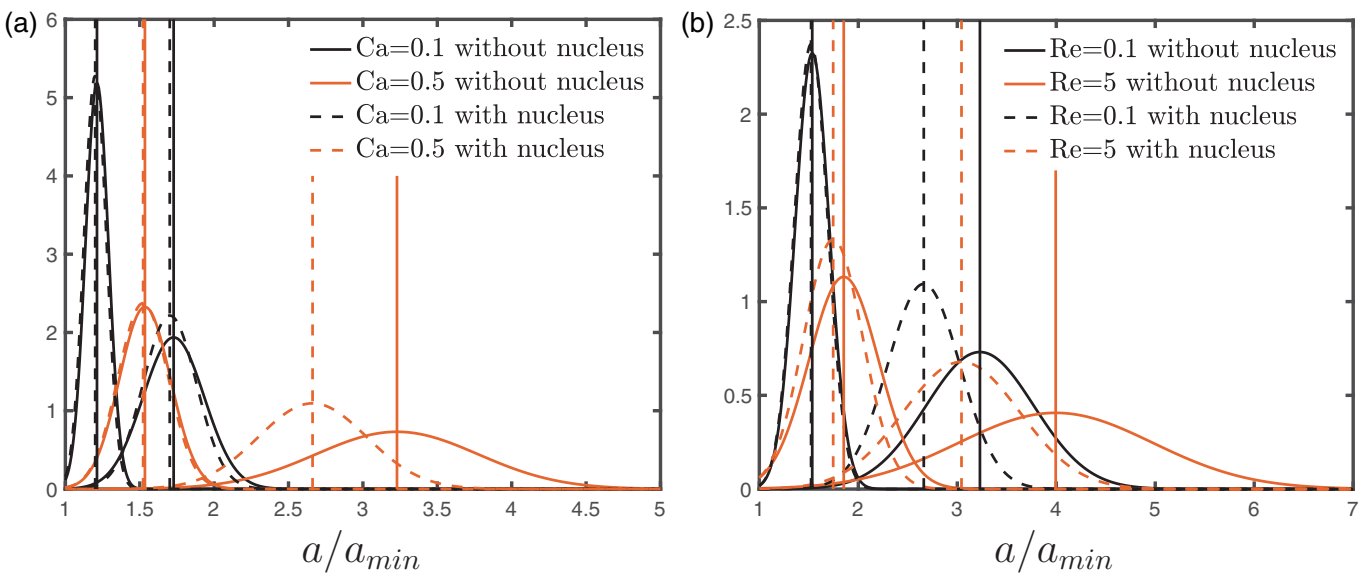

FIG. 5. PDF of the two largest values of the equivalent ellipsoid semiaxes divided by their minimum value for (a) $\mathrm{Re}=0.1$ and different capillary numbers; (b) $\mathrm{Ca}=0.5$ and different Reynolds numbers. The vertical lines represent the mean values.

an indication of the elongation of the capsule, whereas the smallest one provides information about the shape, being 1 for a prolate spheroid and equal to the largest for an oblate.

When varying the capillary number at negligible inertia, $\mathrm{Re}=0.1$, panel (a) in the figure, we observe larger ratios at higher capillary numbers, indicating an average larger deformation of the capsules. Moreover, at $\mathrm{Ca}=0.1$ the distribution of the capsule semiaxes has a more distinct peak, revealing a relatively more uniform distribution of the capsule shape. For this value of the capillary number, the smallest semiaxes ratio is not far from 1 , whereas the largest lies in the range $1 \leqslant$ $a / a_{\min } \leqslant 2.5$, meaning that the equivalent ellipsoids have a prolate shape, with a non-negligible deformation. At $\mathrm{Ca}=0.5$, the range of the semiaxes ratio covered by the capsule in suspensions is much wider and reaches higher values, up to 4.5, suggesting a significant elongation; moreover, the distance between the two ratios increases, showing that the capsules deform more in one direction; however, the smaller of the two ratios also increases slightly, so that their cross-sectional area is elliptical in shape, indicating more heterogeneous stretching of the capsules. In the presence of the rigid nucleus, we note a lower average deformation at $\mathrm{Ca}=0.1$. As mentioned above, this is due to the presence of the nucleus, which makes the middle part of the capsule thicker, which appears as a reduction in the capsule deformation. At $\mathrm{Ca}=0.5$, the difference with the simple capsule data becomes larger for the largest ratio, indicating that the effect of the nucleus is more pronounced at higher capillary numbers. This will be discussed further in Fig. 7 when presenting average values from all cases considered.

The role of the Reynolds number on the deformation is reported in Fig. 5(b), which depicts the $\mathrm{PDF}$ of the semiaxes ratios at $\mathrm{Ca}=0.5$ for Reynolds numbers $\mathrm{Re}=0.1$ and 5 . The relatively peaky distribution at negligible inertia, indicating capsules of similar shapes, is lost when increasing the Reynolds number. In this case, we observe a wider distribution of the semiaxes ratios, indicating a more heterogeneous distribution, as well as larger values (up to values of about 6), revealing the presence of highly elongated capsules. To quantify the homogeneity of the capsule deformation and the distribution of the elongation, Fig. 6 displays the root mean square (rms) of the largest semiaxis ratios of the equivalent ellipsoid. The figure shows that the rms value increases with the capillary number and, more importantly, with the Reynolds number. This is in agreement with the visualization of Fig. 3 showing a more heterogeneous distribution in the capsule shape at $\operatorname{Re}=5$. Finally, it can be inferred from Fig. 6 that the elongation distribution is more uniform for the capsules with a nucleus, which can be a result of the lower freedom of the nucleated capsules for deformation, in particular in their middle part. 


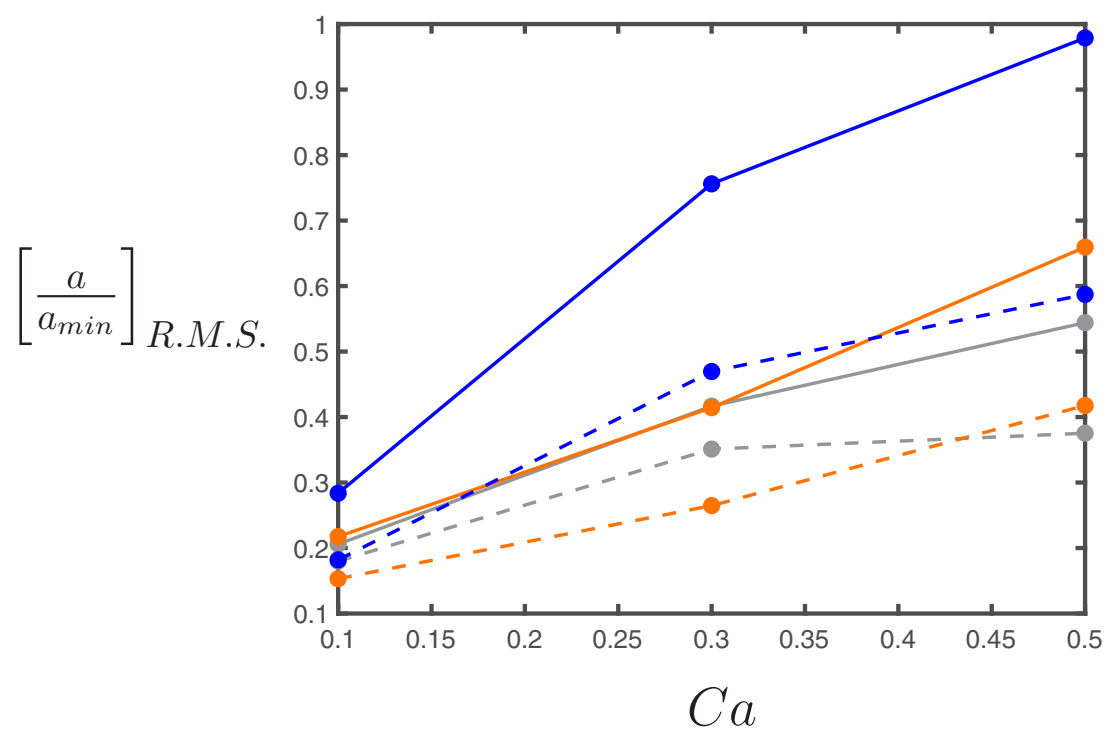

FIG. 6. Maximum root-mean-square values of the largest ratio between semiaxes of the equivalent ellipsoid for different capillary and Reynolds numbers. Solid lines correspond to capsules without a nucleus, and dashed lines represent capsules with a rigid nucleus. Gray, orange, and blue pertain to cases with $\operatorname{Re}=0.1,1$, and 5 .

Further examining the capsule deformation, we present in Fig. 7 the average Taylor parameter and area ratio AR, where the \langle\rangle denote the average over time and the capsules. Figures 7 (a) and 7 (c) clearly show increasing of the capsule deformation with increasing capillary number, which we attribute to larger viscous than elastic forces. The figure also clearly shows that the capsule membrane deforms less in the cases with a nucleus, which we relate to the increase in the capsule thickness in the vicinity of the nucleus [20]. The difference in deformation between capsules with and without a nucleus, as measured by the mean Taylor parameter and the area ratio, increases with the capillary number, confirming that the effect of the nucleus is more pronounced for more deformable membranes. With regard to the effect of inertia, we observe small changes in the Taylor parameter and the area ratio when increasing the Reynolds number from $\operatorname{Re}=0.1$ to 1 , and more significant variations at $\mathrm{Re}=5$ due to the larger drag force on the capsules. Also, the effect of inertia increases with the capillary number, see especially the Taylor parameter, since inertia can increase more easily capsule elongation at larger $\mathrm{Ca}$. The changes of the mean Taylor parameter and area ratio with $\mathrm{Ca}$ at fixed Reynolds number, $\mathrm{Re}=1$, are displayed in Figs. 7(b) and 7(d). The presence of additional simulation points in these figures shows more clearly the increase of the capsule deformation with the capillary number as well as the larger difference in deformation of the simple and nucleated capsules when decreasing the membrane shear modulus, i.e., larger Ca.

As mentioned earlier, we define the orientation of the capsules using the eigenvector of the moment of inertia tensor corresponding to the minimum eigenvalue, i.e., the direction with maximum capsule elongation. The orientation with respect to the flow direction is found by projecting this eigenvector onto the shear plane and computing the angle formed with the streamwise axis. Note that this is a useful measure if the $x$-component of the unit vector $\mathbf{d}$ is larger than the $z$-component, which is the case for many of the configurations investigated here, as shown later. As discussed in $[2,16]$, the orientation with the flow direction has a clear role in determining the suspension rheological properties at negligible inertia, i.e., for the capsule suspensions without a nucleus, the relative viscosity increases with the mean orientation angle in the shear plane.

The PDF of the capsule orientation angle is depicted in Fig. 8 for different capillary and Reynolds numbers. According to panel (a), at negligible inertia the orientation angle decreases 

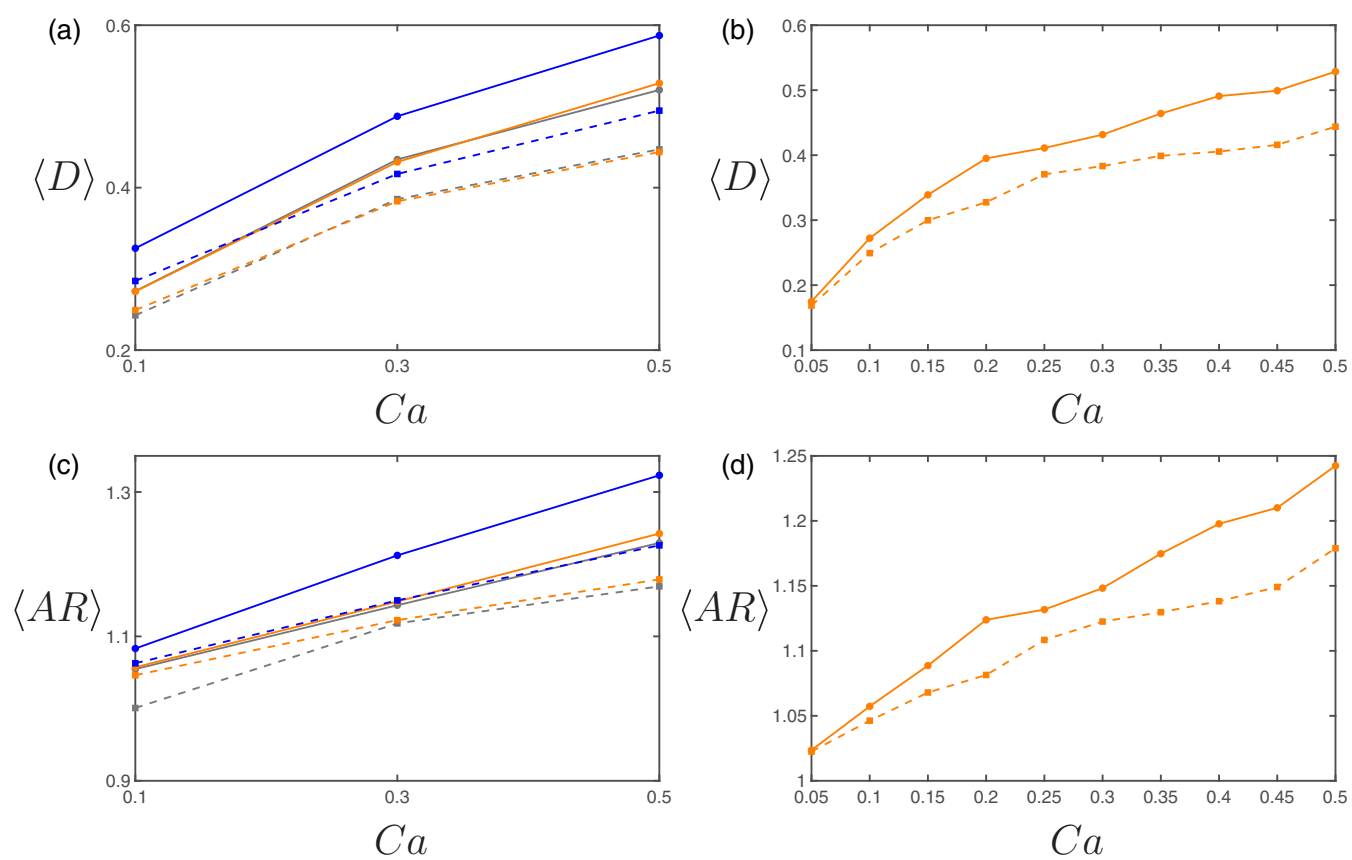

FIG. 7. (a) Mean Taylor parameter and (c) mean area ratio as a function of Re and Ca for volume fraction $\phi=0.31$. Solid lines correspond to capsules without a nucleus, and dashed lines indicate the results for capsules with a nucleus. Gray, orange, and blue denote $\mathrm{Re}=0.1,1$, and 5, respectively. (b) Mean Taylor parameter and (d) mean area ratio at $\mathrm{Re}=1$ with more simulation points for different $\mathrm{Ca}$.

when increasing the capillary number: at higher capillary numbers, the shear forces, which tend to align the capsules with the flow direction, are larger than the elastic restoring forces. Panel (b) of the same figure displays an increase toward large values of the angle as the Reynolds number is increased from 0.1 to 5 . This suggests that the alignment is partially lost when increasing the flow inertia, and it reflects the fact that some elongated capsules tend to orient in the spanwise direction,

(a)

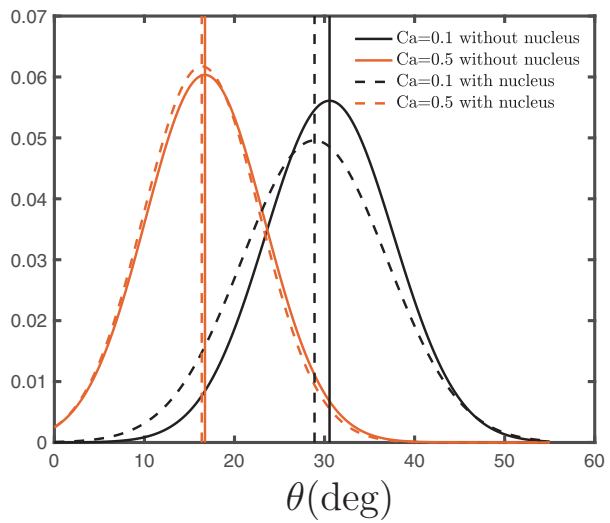

(b)

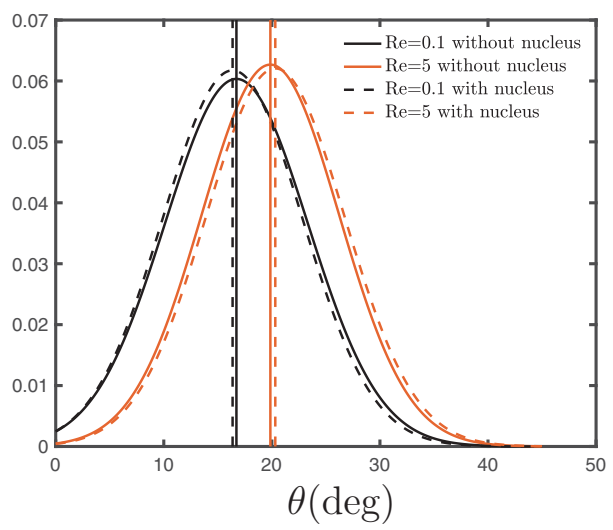

FIG. 8. PDF of the capsule orientation with respect to the streamwise direction for (a) $\operatorname{Re}=0.1$ and different capillary numbers; (b) $\mathrm{Ca}=0.5$ and different Reynolds numbers. See the main text for the exact definition of the orientation angle. The vertical lines represent the mean values. 
(a)

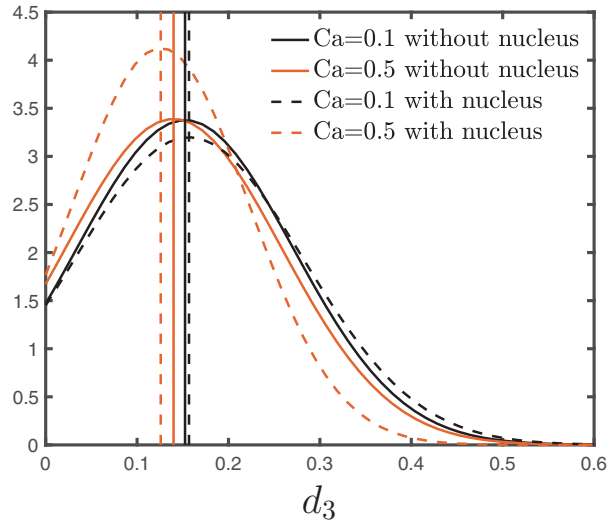

(b)

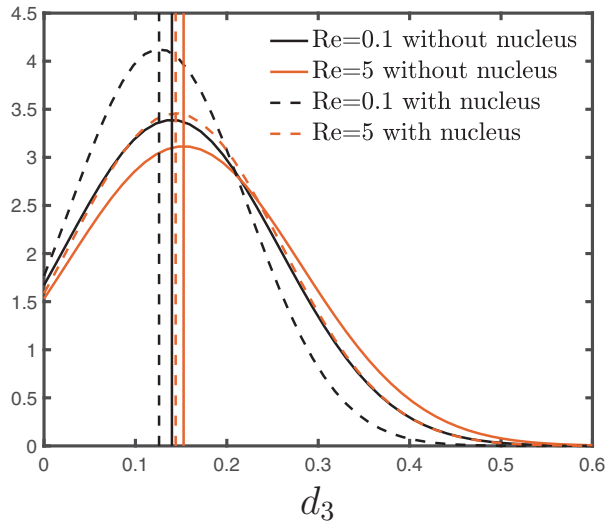

FIG. 9. PDF of the spanwise component of the direction vector, $d_{3}$, for (a) $\operatorname{Re}=0.1$ and different capillary numbers, and (b) $\mathrm{Ca}=0.5$ and different Reynolds numbers. The vertical lines represent the mean values.

as discussed next. Finally, it can be seen that the presence of the nucleus does not have a significant effect on the orientation distributions.

We next study the spanwise orientation of the capsules, quantified by the spanwise component, $d_{3}$, of the unit vector $\mathbf{d}$ parallel to the smallest eigenvalue of the moment of inertia tensor. The PDF of this scalar quantity is displayed in Fig. 9: panel (a) shows that capsules tend to stay longer in the shear plane for higher capillary numbers, i.e., larger spanwise orientations at lower Ca. Panel (b) of the same Fig. 9, instead, shows that the spanwise orientation increases with the Reynolds number so that it is possible to find elongated capsules aligned with the vorticity direction in this case. Moreover, the distribution is wider for $\mathrm{Re}=5$.

The average values of the two orientations are presented in Figs. 10(a)-10(d) for all cases investigated. The data in the figure confirm the trend deduced by the PDF just discussed. We note in addition that the effect of the rigid nucleus on the shear plane orientation is more pronounced at $\mathrm{Ca}=0.1$ while smaller differences are observed when the capsules deform more easily. The additional analysis performed at $\operatorname{Re}=1$, see Figs. 10(b) and 10(d), shows more clearly the alignment with the streamwise direction when increasing the capillary number.

To explain the suspension global behavior, we next consider the different contributions to the average total stress, as introduced in Sec. III B. This budget is presented in Fig. 11, where the stresses are made nondimensional by the reference viscous stress $(\mu \dot{\gamma})$. The viscous stress is always about unity, and the changes in the relative viscosity arise from the membrane and rigid particle (nucleus) stresses. The membrane stress decreases with capillary number; more deformable particles have lower elastic stresses, reflecting the shear thinning with deformability of the suspensions. Comparing the cases with and without a nucleus at $\mathrm{Re}=0.1$ shows that the average elastic stresses are higher for the capsules without a nucleus, which are associated with higher deformations (see Fig. 7). Moreover, the elastic stresses increase when increasing the Reynolds number from 0.1 to 5 , causing the higher shear viscosity discussed above (see Fig. 4). The stresses from the nucleus increase with the membrane capillary number from $\mathrm{Ca}=0.1$ to 0.3 ; this can be related to a decreasing distance between nucleus and membrane when increasing deformability, which increases the viscous shear stress on the rigid nucleus and thus its rotation rate. Further increasing from $\mathrm{Ca}=0.3$ to 0.5 does not change significantly the contribution of the nucleus since there is no further significative change in the shape and size of the gap between the capsule and its nucleus. At a fixed capillary number, the nucleus stresses increase with the Reynolds number, which we associate with stronger forces on a sphere at higher Reynolds numbers; however, this increase is not as large as the increase in the elastic forces. 

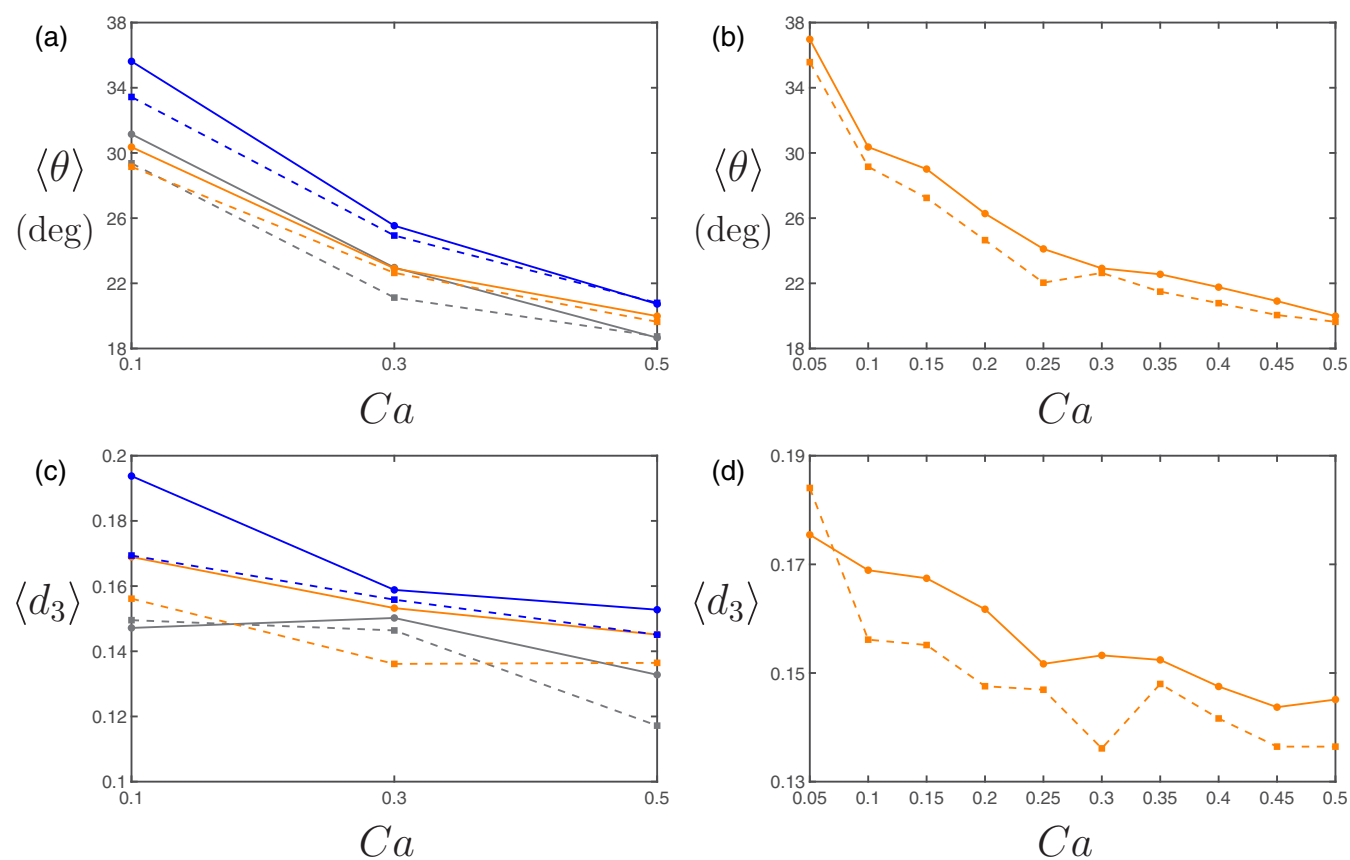

FIG. 10. (a) Mean capsule orientation with respect to the flow direction and (c) mean spanwise component of the principal direction of the momentum of inertia tensor associated with the minimum eigenvalue (direction of maximum elongation) as a function of the Reynolds number Re and capillary number $\mathrm{Ca}$ for volume fraction $\phi=0.31$. Solid lines correspond to capsules without a nucleus, and dashed lines indicate the results for capsules with nucleus. Gray, orange, and blue denote $\operatorname{Re}=0.1,1$, and 5, respectively. (b) Mean capsule orientation and (d) mean spanwise component of the direction of maximum elongation at $\operatorname{Re}=1$ for several values of $\mathrm{Ca}$.

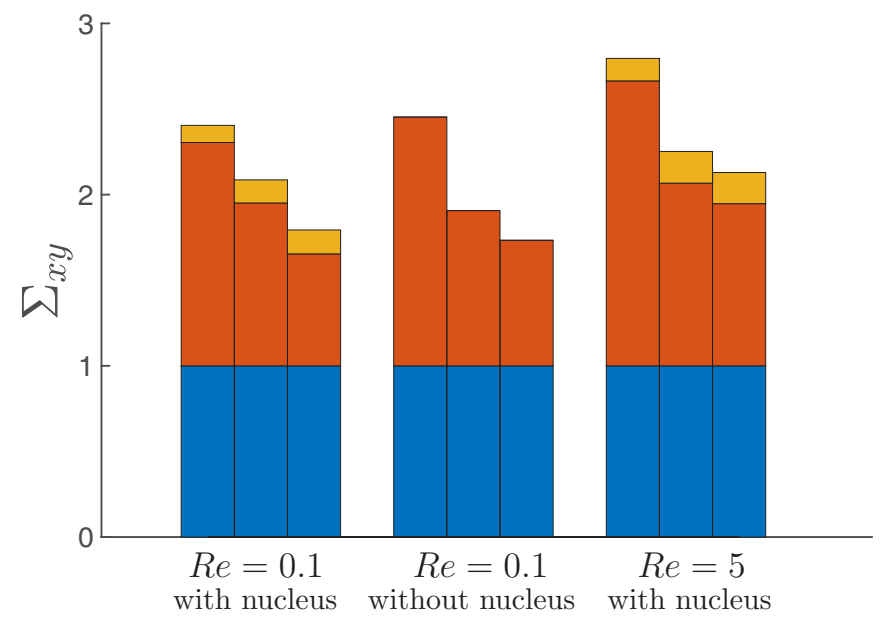

FIG. 11. Budget of stress for the nucleated-capsule suspensions with different Reynolds and capillary number and volume fraction $\phi=0.31$. Blue denotes the fluid flow contribution, red represents the contribution of the elastic forces, and orange denotes the contribution of the rigid nucleus to the total shear stress. 

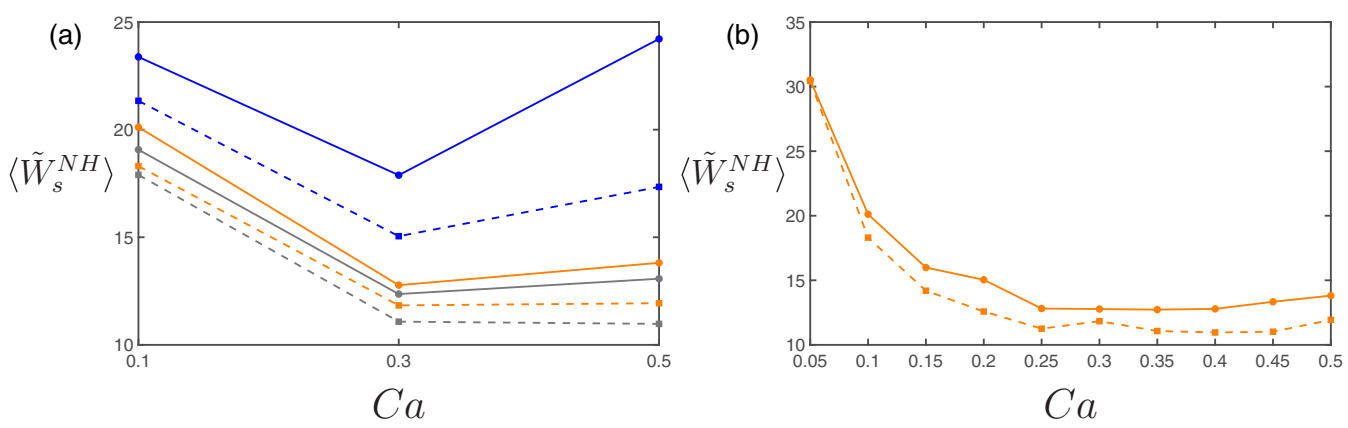

FIG. 12. (a) Mean capsule elastic energy as a function of the Reynolds number, Re, and capillary number, $\mathrm{Ca}$, for volume fraction $\phi=0.31$. Solid lines correspond to capsules without a nucleus, and dashed lines to capsules with a nucleus. Gray, orange, and blue denote different Reynolds numbers, $\operatorname{Re}=0.1,1$, and 5 . (b) Mean capsule elastic energy at $\mathrm{Re}=1$ over a wider range of $\mathrm{Ca}$.

Next, we examine the mean elastic energy of the suspension, normalized with the viscous scale, defined as the average over all capsules of

$$
\tilde{W}_{s}^{\mathrm{NH}}=\frac{1}{2 \mathrm{Ca}}\left(I_{1}-1+\frac{1}{I_{2}+1}\right)+\frac{2 B}{\mathrm{Ca}} H^{2},
$$

where $H$ is the mean local curvature, and the invariants $I_{1}$ and $I_{2}$ have been defined above. The results are presented in Fig. 12. The data in the plot indicate that, for a fixed capillary number, the mean elastic energy increases when increasing the Reynolds number, i.e., increasing inertial effects, due to the larger deformations of the capsule membrane. The largest bending energy is attained for the case of simple capsules with the highest Reynolds and capillary numbers considered in this study. Examining the data at a fixed Reynolds number, we observe a nonmonotonic dependence of the elastic energy on the capillary number, with a minimum at intermediate values of $\mathrm{Ca}$, followed by a plateau or a weak increase when inertia is relevant. This minimum appears to move toward larger values of $\mathrm{Ca}$ when decreasing the Reynolds number. The elastic energy is proportional to the inverse of the capillary number and to the deformation of the membrane, which increases with the $\mathrm{Ca}$. This combined effect results in a nonmonotonic behavior as shown more clearly in Fig. 12(b) for $\operatorname{Re}=1$, where we display data over a wider range of capillary numbers. Finally, we note that the elastic energy is always less for nucleated capsules than for the simple ones, which we explain with their smaller deformation.

To quantify the viscoelasticity of the suspensions, we last examine the first normal stress difference, introduced above in Eq. (26). Figure 13 depicts the average first normal stress as a function of the capillary number for simple and nucleated capsules and the different Reynolds numbers under investigation. At a fixed Reynolds number, we mainly observe an increase of the first normal stress difference with the capillary number due to larger deformation and more alignment in the shear plane (see Figs. 7 and 10), which results in larger anisotropy of the stress tensor [2]. For capsules without nucleus, the normal stress difference slightly reduces from $\mathrm{Ca}=0.3$ to 0.5 at $\mathrm{Re}=0.1$, which is consistent with the reduction of mean elastic energy reported in Fig. 12. This may likely be attributed to a sharper increase in the average wall-normal stress due to the capsule-capsule interactions. The inertial effects (varying Reynolds number) are highly dependent on the capillary number since $N_{1}$ depends both on streamwise and wall normal stresses, which depend differently on the deformed capsule shape. In general, the nucleated capsule suspensions have a lower first normal stress difference than simple capsule suspensions due to lower capsule deformation, as is clearly shown in Fig. 13(b) at $\mathrm{Re}=1$. As a consequence of the presence of a nucleus, $N_{1}$ is less sensitive to the Reynolds number in nucleated suspensions. In fact, the first normal stress difference 

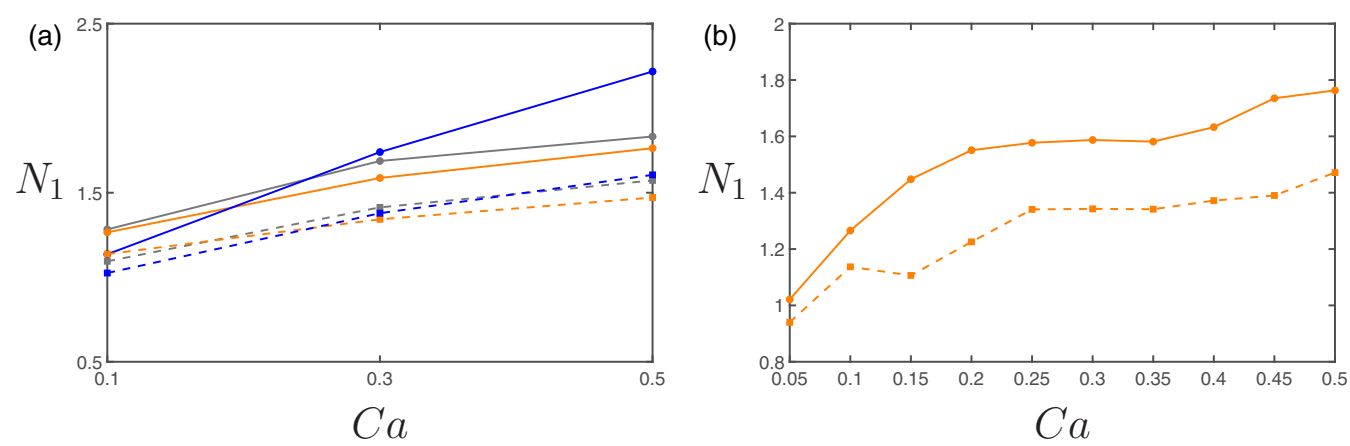

FIG. 13. (a) First normal stress difference as a function of Re and Ca for volume fraction $\phi=0.31$. Solid lines correspond to capsule without a nucleus, whereas dashed lines represent results for capsules with a nucleus. Gray, orange, and blue denote $\mathrm{Re}=0.1,1$, and 5. (b) First normal stress difference over a wider range of $\mathrm{Ca}$ for $\mathrm{Re}=1$.

is a result of the capsule anisotropy in the shear plane, and the presence of a rigid nucleus reduces this anisotropy.

\section{B. Effect of volume fraction}

In this section, we study the effect of the capsule volume fraction on the rheological properties of the suspensions and the capsule average motion and deformation. We consider two capillary numbers, $\mathrm{Ca}=0.1$ and 0.3 , and two Reynolds numbers, $\mathrm{Re}=1$ and 5 . The relative suspension viscosity is depicted in Fig. 14, whereas the average capsule properties-the Taylor parameter, the area ratio, the angle between the axis of largest elongation and the streamwise direction, and the spanwise component of the unit vector parallel to this axis orientation-are presented in Fig. 15.

As expected, the relative viscosity increases with the volume fraction, due to the increased forces from the suspended objects back to the flow. The rate of increase of the relative viscosity is higher for $\mathrm{Ca}=0.1$, in agreement with the observations of Matsunaga et al. [2]. The difference between the relative viscosity of the sample and the nucleated capsule suspensions is secondary in comparison to the effect of volume fraction, similar to the case of deformable objects when variations of the capillary number are less relevant than variations of the volume fraction $[14,15,41]$; in this particular

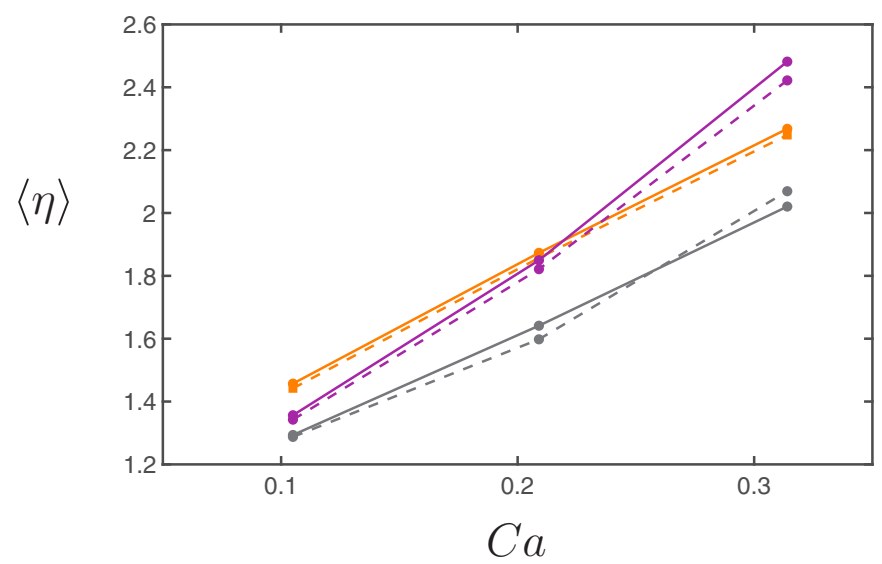

FIG. 14. Relative viscosity of nucleated and denucleated capsules vs the volume fraction $\phi$. Solid and dashed lines represent capsules with and without a nucleus, whereas gray and orange indicate flows with $\mathrm{Re}=1$ and 5 at $\mathrm{Ca}=0.3$; the purple lines show the results for $\mathrm{Re}=1$ and $\mathrm{Ca}=0.1$. 

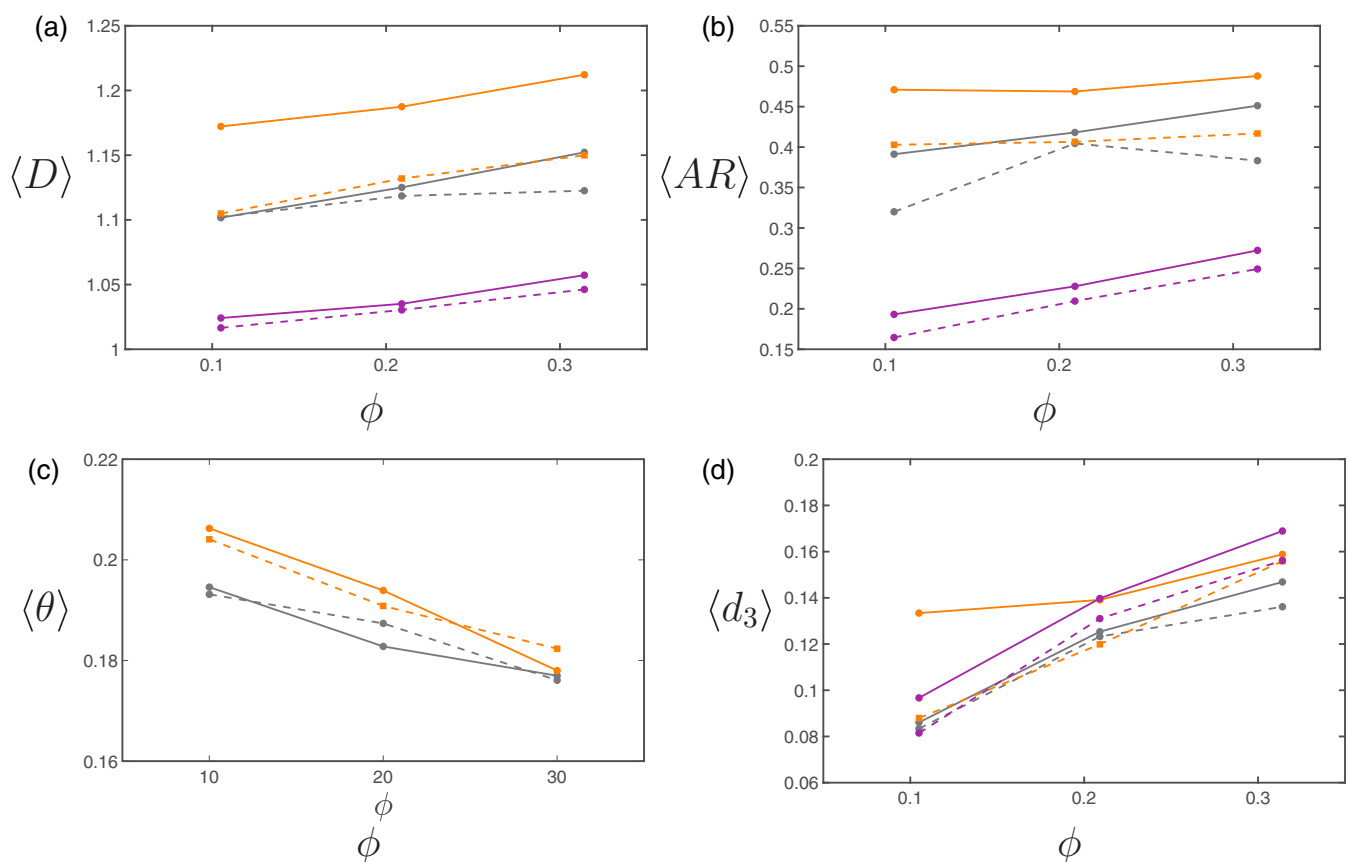

FIG. 15. Averaged capsule deformation measured in terms of (a) the Taylor parameter and (b) area ratio. Particle orientation measured by (c) the average angle between the projection in the shear plane of the axis of the largest capsule elongation and the streamwise direction for $\mathrm{Ca}=0.3$, and (d) average spanwise component of the unit vector parallel to the axis of maximum elongation. Solid and dashed lines represent capsules with and without a nucleus, whereas gray and orange indicate flows with $\mathrm{Re}=1$ and 5 and $\mathrm{Ca}=0.3$. The purple lines show the results for $\mathrm{Re}=1$ and $\mathrm{Ca}=0.1$.

case, this can be the result of different effects having the opposite impact on the relative viscosity: the capsule deformation, orientation angle, and the presence of the rigid nucleus. The relative viscosity increases with the mean capsule deformation and the orientation angle (lower alignment with the flow direction) [2]; in addition, the stresses due the nucleus tend to increase the relative viscosity. For a fixed capillary and Reynolds number, when adding a nucleus, these three effects can balance each other as particle deformation is reduced [see Figs. 15(a)-15(c)], resulting in small variations of the effective viscosity, as shown in Fig. 14.

Figures 15(a) and 15(b) show that the capsule deformation mainly increases with the volume fraction due to the larger shear between the capsules. There is a slight reduction in the area ratio with the volume fraction at $\mathrm{Ca}=0.3$ and $\mathrm{Re}=1$ which can be attributed to the larger normal forces on the capsule membrane from the adjacent capsules. The angle between the projection in the shear plane of the axis of largest capsule elongation and the streamwise direction is displayed in Fig. 15(c), revealing a reduction in the orientation angle, i.e., a stronger average alignment, with the volume fraction due to larger shear forces between the capsules. The spanwise component of the capsule orientation, depicted in Fig. 15(d), displays an increase with the volume fraction and a more disordered microstructure (yet with the axis of elongation almost orthogonal to the wall-normal direction, due to increased capsule-capsule interactions).

Figure 16, left panel, represents the first normal stress difference as a function of the volume fraction, indicating an increase in viscoelasticity with the volume fraction. The increase is due to increasing elastic forces as well as a decrease of the mean orientation angle with the volume fraction (see Fig. 15), which adds more anisotropy to the flow; see also Ref. [2]. The data in the figure also show that, at constant Reynolds number, the first normal stress difference is lower for the nucleated 

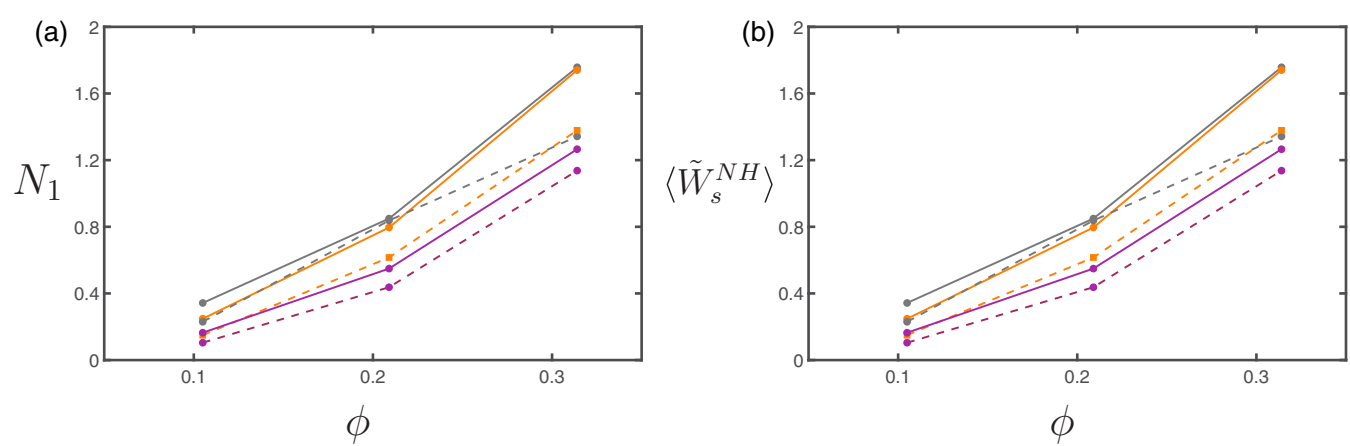

FIG. 16. (a) First normal stress difference and (b) elastic energy vs the capsule volume fraction. Solid and dashed lines represent capsules with and without a nucleus, whereas gray and orange indicate flows with $\mathrm{Re}=1$ and 5 with $\mathrm{Ca}=0.3$. The purple lines show the results for $\mathrm{Re}=1$ and $\mathrm{Ca}=0.1$.

capsules than for the simple capsules due to the lower deformation in the presence of a nucleus. Finally, it can be observed that the effect of the presence of a nucleus on the first normal stress difference is more pronounced than on the relative viscosity. Finally, the mean elastic energy of the capsules is shown in Fig. 16(b) versus the volume fraction. Here, we can see that the mean capsule elastic energy increases with the volume fraction and the Reynolds number, due to the increased deformation of the capsules, in agreement with the results in Fig. 15. The increased deformation is the result of stronger capsule-capsule interactions at higher volume fractions. Examining the data at constant Reynolds number, we note that the elastic energy is lower for the nucleated capsule suspensions, which reflects the lower deformation of the capsule membranes in the presence of a rigid nucleus.

\section{CONCLUSIONS}

We present the results of interface-resolved numerical simulations of suspensions of initially spherical capsules with and without a rigid nucleus inside. The capsule is modeled as a thin hyperelastic membrane enclosing a droplet of a second fluid, which is assumed here to have the same density and viscosity as the outer ambient fluid. Continuum elasticity equations are solved for the membrane assuming a Neo-Hookean material with the fluid and solid motions coupled via an immersed boundary method. To model the presence of a nucleus, we add a rigid particle with radius equal to half the initial radius of the capsule and located initially at the center of each capsule. A series of simulations is performed to study the effect of capillary number, i.e., membrane deformability, Reynolds number, i.e., inertial effects, and volume fraction on the global suspension behavior for capsules with and without a nucleus. In particular, we report the relative viscosity and the first normal stress difference of the different suspensions considered, and we try to relate the macroscopic system behavior to the capsule deformation, orientation in the shear plane, degree of alignment in the spanwise direction, and elastic energy. The stress budget for suspensions of different capillary and Reynolds numbers is also presented.

The results indicate that, at a constant Reynolds number, the relative viscosity of the suspensions decreases with the capillary number, so the suspension is shear thinning with respect to deformability, as observed in previous studies on suspensions of flexible and deformable objects. For the range of parameters investigated, the effect of the flow inertia becomes evident when increasing the Reynolds number from 1 to 5 , leading to an increase of the relative viscosity. Inertial effects are minimal for $\operatorname{Re} \in[0.1,1]$. The capsule deformation increases with inertia and decreases in the presence of a rigid nucleus; in particular, the presence of the nucleus reduces the capsule mean deformation in all cases considered. The alignment with the flow is found to depend mainly on the capillary number and to increase with the Reynolds number. The presence of the nucleus is more 
apparent for the stiffer membrane investigated here, $\mathrm{Ca}=0.1$, and decreases at higher capillary numbers.

The first normal stress difference, used to quantify the viscoelastic behavior of the system, is always lower for the nucleated capsules and increases with the capillary number. The variations of the first normal stress difference with the Reynolds number are found to depend on the capillary number. At a fixed capillary number, the mean elastic energy is always lower for a nucleated capsule than for those without a nucleus, as explained by the reduced deformation of the former, and it increases with the Reynolds number. The elastic energy varies nonmonotonically with the capillary number as a result of two counteracting effects, namely the proportionality to the inverse of the capillary number and to the capsule deformation, which increases with $\mathrm{Ca}$.

When varying the capsule volume fraction, the relative viscosity and first normal stress increase with the number of capsules, the effect of the nucleus on first normal stress difference being more than that on the relative viscosity. The capsule alignment with the flow direction decreases with the volume fractions due to increased capsule-capsule interactions. Note that we have kept constant the viscosity contrast between inner and outer fluid, as well as the ratio between nucleus and capsule size. The analysis of these effects is left as future extensions of this first work. Moreover, the analysis performed here quantifies the viscoelastic behavior of the suspension under constant shear. To gain further insight on this aspect, the system response to small and large amplitude oscillations should also be an object of future work.

[1] D. A. Reasor, J. R. Clausen, and C. K. Aidun, Rheological characterization of cellular blood in shear, J. Fluid Mech. 726, 497 (2013).

[2] D. Matsunaga, Y. Imai, T. Yamaguchi, and T. Ishikawa, Rheology of a dense suspension of spherical capsules under simple shear flow, J. Fluid Mech. 786, 110 (2016).

[3] J. Gounley, E. W. Draeger, and A. Randles, Numerical simulation of a compound capsule in a constricted microchannel, Proc. Comput. Sci. 108, 175 (2017).

[4] D. Barthes-Biesel and V. Chhim, The constitutive equation of a dilute suspension of spherical microcapsules, Int. J. Multiphase Flow 7, 493 (1981).

[5] P. Snabre and P. Mills, Rheology of concentrated suspensions of viscoelastic particles, Colloids Surf. A 152, 79 (1999).

[6] R. M. MacMeccan, J. R. Clausen, G. P. Neitzel, and C. K. Aidun, Simulating deformable particle suspensions using a coupled lattice-boltzmann and finite-element method, J. Fluid Mech. 618, 13 (2009).

[7] P. Bagchi and R. M. Kalluri, Rheology of a dilute suspension of liquid-filled elastic capsules, Phys. Rev. E 81, 056320 (2010).

[8] P. Bagchi and R. M. Kalluri, Dynamic rheology of a dilute suspension of elastic capsules: effect of capsule tank-treading, swinging and tumbling, J. Fluid Mech. 669, 498 (2011).

[9] J. R. Clausen, D. A. Reasor, and C. K. Aidun, The rheology and microstructure of concentrated noncolloidal suspensions of deformable capsules, J. Fluid Mech. 685, 202 (2011).

[10] T. Krüger, B. Kaoui, and J. Harting, Interplay of inertia and deformability on rheological properties of a suspension of capsules, J. Fluid Mech. 751, 725 (2014).

[11] M. Gross, T. Krüger, and F. Varnik, Rheology of dense suspensions of elastic capsules: normal stresses, yield stress, jamming and confinement effects, Soft Matter 10, 4360 (2014).

[12] D. A. Fedosov, H. Noguchi, and G. Gompper, Multiscale modeling of blood flow: from single cells to blood rheology, Biomech. Model. Mechanobiol. 13, 239 (2014).

[13] R. G. Winkler, D. A. Fedosov, and G. Gompper, Dynamical and rheological properties of soft colloid suspensions, Curr. Opin. Colloid Interface Sci. 19, 594 (2014).

[14] M. E. Rosti and L. Brandt, Suspensions of deformable particles in a couette flow, J. Non-Newtonian Fluid Mech. (c) 262, 3 (2018).

[15] M. E. Rosti, L. Brandt, and D. Mitra, Rheology of suspensions of viscoelastic spheres: Deformability as an effective volume fraction, Phys. Rev. Fluids 3, 012301(R) (2018). 
[16] N. Takeishi, M. E. Rosti, Y. Imai, S. Wada, and L. Brandt, Hemorheology in dilute, semi-dilute and dense suspensions of red blood cells, J. Fluid Mech. 872, 818 (2019).

[17] Y. Imai, H. Kondo, T. Ishikawa, C. T. Lim, and T. Yamaguchi, Modeling of hemodynamics arising from malaria infection, J. Biomech. 43, 1386 (2010).

[18] Z. Y. Luo, L. He, and Bo. F. Bai, Deformation of spherical compound capsules in simple shear flow, J. Fluid Mech. 775, 77 (2015).

[19] Z. Y. Luo and Bo. F. Bai, Dynamics of nonspherical compound capsules in simple shear flow, Phys. Fluids 28, 101901 (2016).

[20] A. Alizad Banaei, J.-C. Loiseau, I. Lashgari, and L. Brandt, Numerical simulations of elastic capsules with nucleus in shear flow, Eur. J. Comput. Mech. 26, 131 (2017).

[21] C. Pozrikidis, Effect of membrane bending stiffness on the deformation of capsules in simple shear flow, J. Fluid Mech. 440, 269 (2001).

[22] C. Pozrikidis, Computational Hydrodynamics of Capsules and Biological Cells (CRC, Boca Raton, FL, 2010).

[23] H. Zhao, A. H. G. Isfahani, L. N. Olson, and J. B. Freund, A spectral boundary integral method for flowing blood cells, J. Comput. Phys. 229, 3726 (2010).

[24] M. N. Ardekani, P. Costa, W. P. Breugem, and L. Brandt, Numerical study of the sedimentation of spheroidal particles, Int. J. Multiphase Flow 87, 16 (2016).

[25] F. Picano, W.-P. Breugem, and L. Brandt, Turbulent channel flow of dense suspensions of neutrally buoyant spheres, J. Fluid Mech. 764, 463 (2015).

[26] I. Lashgari, F. Picano, W. P. Breugem, and L. Brandt, Channel flow of rigid sphere suspensions: particle dynamics in the inertial regime, Int. J. Multiphase Flow 78, 12 (2016).

[27] C. S. Peskin, Flow patterns around heart valves: a digital computer method for solving the equations of motion, IEEE Trans. Biomed. Eng. 4, 316 (1973).

[28] R. Mittal and G. Iaccarino, Immersed boundary methods, Annu. Rev. Fluid Mech. 37, 239 (2005).

[29] A. Roma, C. Peskin, and M. Berger, An adaptive version of the immersed boundary method, J. Comput. Phys. 153, 509 (1999).

[30] P. Pranay, S. G. Anekal, J. P. Hernandez-Ortiz, and M. D. Graham, Pair collisions of fluid-filled elastic capsules in shear flow: Effects of membrane properties and polymer additives, Phys. Fluids 22, 123103 (2010).

[31] W.-P. Breugem, A second-order accurate immersed boundary method for fully resolved simulations of particle-laden flows, J. Comput. Phys. 231, 4469 (2012).

[32] I. Lashgari, F. Picano, W.-P. Breugem, and L. Brandt, Laminar, Turbulent, and Inertial Shear-Thickening Regimes in Channel Flow of Neutrally Buoyant Particle Suspensions, Phys. Rev. Lett. 113, 254502 (2014).

[33] Y. Liu, L. Zhang, X. Wang, and W. K. Liu, Coupling of navier-stokes equations with protein molecular dynamics and its application to hemodynamics, Int. J. Numer. Methods Fluids 46, 1237 (2004).

[34] D. Alghalibi, I. Lashgari, L. Brandt, and S. Hormozi, Interface-resolved simulations of particle suspensions in newtonian, shear thinning and shear thickening carrier fluids, J. Fluid Mech. 852, 329 (2018).

[35] F. Picano, W.-P. Breugem, D. Mitra, and L. Brandt, Shear Thickening in Non-Brownian Suspensions: An Excluded Volume Effect, Phys. Rev. Lett. 111, 098302 (2013).

[36] G. K. Batchelor, The stress system in a suspension of force-free particles, J. Fluid Mech. 41, 545 (1969).

[37] J. R. Taylor et al., Classical Mechanics (University Science Books, Sausalito, 2005).

[38] S. Ramanujan and C. Pozrikidis, Deformation of liquid capsules enclosed by elastic membranes in simple shear flow: large deformations and the effect of fluid viscosities, J. Fluid Mech. 361, 117 (1998).

[39] S. B. Lindström and T. Uesaka, Simulation of semidilute suspensions of non-brownian fibers in shear flow, J. Chem. Phys. 128, 024901 (2008).

[40] L. H. Switzer III and D. J. Klingenberg, Rheology of sheared flexible fiber suspensions via fiber-level simulations, J. Rheol. 47, 759 (2003).

[41] A. A. Banaei, M. E. Rosti, and L. Brandt, Numerical study of filament suspensions at finite inertia, J. Fluid Mech. 882, A5 (2020). 